\title{
Visual hallucinations in Lewy body disease: pathophysiological insights from phenomenology
}

\author{
Fabrizia D'Antonio ${ }^{1}$ - Maddalena Boccia ${ }^{2,3} \cdot$ Antonella Di Vita $^{1} \cdot$ Antonio Suppa $^{1,4} \cdot$ Andrea Fabbrini $^{4}$. \\ Marco Canevelli ${ }^{1}$. Francesca Caramia ${ }^{1}$. Marco Fiorelli ${ }^{1}$. Cecilia Guariglia ${ }^{2,3}$. Stefano Ferracuti ${ }^{1} \cdot$ Carlo de Lena $^{5}$. \\ Dag Aarsland ${ }^{6} \cdot$ Dominic ffytche $^{6}$
}

Received: 6 November 2021 / Revised: 16 January 2022 / Accepted: 19 January 2022 / Published online: 31 January 2022

(c) The Author(s) 2022

\begin{abstract}
Visual hallucinations (VH) in Lewy body disease (LBD) have a heterogenous phenomenology classified into minor phenomena (MVH) and complex hallucinations (CVH). Mechanisms underpinning VH and their temporal aspects are largely unknown. According to the hodotopic model, we investigated whether changes in distinct cognitive domains and neural networks in the hallucination trait underpin temporal aspects of $\mathrm{MVH}$ and $\mathrm{CVH}$ in the hallucination state. $35 \mathrm{LBD}$ patients with VH underwent a complete neuropsychological evaluation and resting-state fMRI. North-East-Visual-HallucinationsInterview was used to assess their typical $\mathrm{VH}$ content, duration, and frequency. We found that MVH was not associated with cognitive impairment, while $\mathrm{CVH}$ was associated with impairments in visuoperceptual processes, attention and visual abstract reasoning. In seed-to-seed functional connectivity (FC) analysis we identified functional couplings associated with $\mathrm{MVH}$ and CVH temporal severity (duration $\mathrm{x}$ frequency), duration and frequency. MVH severity was negatively associated with FC between early visual areas (EVA) and ventral-visual-stream regions, and negatively associated with FC between brainstem and EVA, which may be linked to LBD brainstem neuropathology. CVH duration was positively associated with FC between ventral-visual stream and salience network (SN). CVH frequency was negatively associated with FC between DMN and SN. Functional alterations in distinct visual and attentional networks and their dynamic interaction in trait LBD hallucinators are linked to both the phenomenology of state content and its temporal characteristics. Within a network, $\mathrm{VH}$ frequency and duration may be linked to different types of functional alterations: increased connectivity leading to sustained activity prolonging VH (duration) and decreased connectivity increasing dysregulated, spontaneous activity (frequency). These findings support the hodotopic hypothesis of VH and may reflect a link between VH phenomenology, LBD neuropathological progression and the involvement of specific neurotransmitter systems.
\end{abstract}

Keywords Visual hallucination $\cdot$ Lewy body dementia $\cdot$ Parkinson dementia $\cdot$ Neuroimaging $\cdot$ Misperceptions

Fabrizia D’Antonio

fabrizia.dantonio@uniroma1.it

1 Department of Human Neuroscience, "Sapienza” University of Rome, Viale dell'Università, 3000165 Rome, Italy

2 Department of Psychology, Sapienza University of Rome, Rome, Italy

3 Cognitive and Motor Rehabilitation Unit, IRCSS Fondazione Santa Lucia, Rome, Italy

4 IRCCS Neuromed Institute, Pozzilli, IS, Italy

5 IRCCS San Raffaele, Rome, Italy

6 Institute of Psychiatry, Psychology and Neuroscience, King's College London, London, UK 


\section{Abbreviations}

AchEI Acetylcholinesterase inhibitors

AG Angular gyrus

AIM Activation-input-modulation

BNT Boston naming test

CDT Clock drawing test

aPaHC Anterior division of parahippocampal gyrus

aSMG Division of supramarginal gyrus

CBT Corsi block tapping test

$\mathrm{CVH} \quad$ Complex visual hallucinations

DA Dopamine agonists

DAN Dorsal attention network

DLB Dementia with Lewy bodies

DMN Default mode network

DS Digit span

EVA Early visual areas

FAB Frontal assessment battery

FC Functional connectivity

iLOC Inferior division of lateral occipital cortex

LB Lewy bodies

LBD Lewy body disease

LG Lingual gyrus

MMSE Mini mental state examination

MVH Minor visual hallucinations

NEVHI North-east visual hallucinations interview

NPI

OFusG

OP

PAD

$\mathrm{PC}$

$\mathrm{PD}$

PDD

PVF

pPaHC Posterior division of parahippocampal gyrus

pSMG Posterior division of supramarginal gyrus

RAVLT Rey's auditory verbal learning test

RCPM Raven's colored progressive matrices

RCFT Rey-osterrieth complex figure test

sLOC Superior division of lateral occipital cortex

SN Salience network

SVF Semantic verbal fluency

TMT-A Trail making test part A

TMT-B Trail-making test part B

TOFusC Temporo occipital fusiform cortex

ToITG Temporo occipital part of inferior temporal gyrus

toMTG Temporo occipital part of middle temporal gyrus

VAN Ventral attention network

UPDRS Unified Parkinson's Disease Rating Scale

VH Visual hallucinations

VS Visual search test

\section{Introduction}

The mechanisms underpinning visual hallucinations in Lewy Body Disease (LBD) are largely unknown. LBD represents a spectrum including Parkinson's disease (PD), Parkinson's disease dementia (PDD), and dementia with Lewy bodies (DLB) [1-3], which share the same neuropathological marker, namely the intracellular inclusion of alpha-synuclein (Lewy bodies (LB)), as well as clinical features such as visual hallucinations (VH). Many theories have been proposed to explain the brain mechanisms underlying these phenomena. In LBD, models focusing on complex VH contents highlight alterations in cognitive and perceptual mechanisms. The Activation-Input-Modulation (AIM) Model, hypothesizes the need of the three factors for the hallucination's onset. An activator factor which could be the level of arousal or an environmental factor such as a low ambient stimulation, an input factor which relies on the gating balance between internal stimulus generation and exterior perceptions, and a modulation factor which is the modulatory effects of the neurotransmitters [4]. According to the Perception and Attention Deficit (PAD) model, VH have their roots in the co-occurrence of visuoperceptual impairment and attentional domain alterations, which results in an unbalance between bottom-up and top-down processing [5]. Furthermore, the Attention and Control Model by Shine and colleagues $[6,7]$ posits that $\mathrm{VH}$ are due to reduced engagement of the dorsal attention network (DAN), associated with a greater engagement of the ventral attention network (VAN), and the intrusion of the default mode network (DMN), resulting in greater salience devoted to the stimuli and intrusion of internal thought respectively. Shine and colleagues provided evidence for this model using fMRI during visual stimulation: PD patients with VH showed decreased activation in the frontal eye field and parietal lobe (part of the DAN), which was associated with increased activity in the DMN and VAN when ambiguous stimuli were presented [8]. Overall, AIM, PAD and Attention and Control Models point out a key role of attention. Previous studies showing attentional impairment in PDD patients [9] and both attentional and verbal learning impairments in PD patients [10] have confirmed this idea. Also in DLB patients, a visual attention deficit has been shown to be closely linked to $\mathrm{VH}$ $[11,12]$. Beyond LBD, the deafferentation-hyperexcitability model of $\mathrm{VH}$ posits that altered excitability in the visual associative cortices due to deafferentation is the main mechanism underpinning the simple and complex $\mathrm{VH}$ of eye and visual pathway disease, as has been demonstrated in the context of Charles Bonnet syndrome [13, 14]

The hodotopic framework provides a functional anatomical context for such models and the mechanisms underlying VH. Thus, it integrates accounts emphasizing the topological 
role of functionally specialized brain regions with accounts highlighting the role of large-scale brain networks. It posits that spontaneous increases in activity in specialized visual cortices at the time of a hallucination (the hallucination state) that defines the content of a VH, occurs in the context of longer-term network alterations (the hallucination trait) which underlie a predisposition towards VH [15]. Only a few studies that mainly focused on the role of the DMN have explored the functional alterations associated with $\mathrm{VH}$ in LBD. In brief, these studies found that DMN activity was higher in PD with VH and reduced in PD without VH [16]. Additionally, PD patients with $\mathrm{VH}$ showed higher functional couplings between specific nodes of the DMN, namely the precuneus, posterior cingulate cortex, and right middle frontal regions, than PD without $\mathrm{VH}$ [17].

The phenomenology of VH in LBD is heterogenous and comprises several types of minor and complex hallucinations. Minor phenomena (MVH) include illusions, presence hallucinations (i.e., the feeling that someone is close by or has just left the room), and passage hallucinations (i.e. the vision of a shadow of something or someone passing fast in the periphery of eye field) $[14,18]$. Complex visual hallucinations $(\mathrm{CVH})$ in the LBD spectrum, consist of well-formed visions of insects, people (familiar or unfamiliar, alive or dead), inanimate objects, animals, animated figures (often children), normal-size figures, or miniature people [19]. More broadly, phenomenology can also be considered to include temporal aspects of $\mathrm{VH}$ such as how long each hallucination lasts and how frequently the hallucinations occur. According to the hodotopic approach, $\mathrm{VH}$ phenomenology can help reveal both localized brain regions and brain networks involved in VH. For example, in the PD spectrum VH phenomenology may track disease progression according to Braak stage [20]. When LBs affect the brain stem, MVH occur due to dysfunction in networks connecting subcortical regions such as the brainstem to cortical visual areas, particularly the dorsal visual stream. At later disease stages when LBs progress toward the forebrain and limbic regions, $\mathrm{CVH}$ appear. $\mathrm{CVH}$ may be either the result of direct involvement of ventral-visual stream regions or the result of cholinergic deafferentation from subcortical regions to ventral-visual stream regions [14].

Despite the high prevalence and associated poor outcomes of CVH and MVH in LBD [14], there are currently no evidence-based medications to treat them or an understanding of the mechanisms underlying how frequently they occur or their duration. A better understanding of the underlying pathophysiology of these temporal phenomenological aspects would potentially point to novel treatment approaches. The aim of the present study was to identify VH neural correlates in LBD from a hodotopic perspective, identifying VH 'trait' network alterations that mark a predisposition to specific contents (whether $\mathrm{CVH}$ or $\mathrm{MVH}$ ) and temporal features (their duration or frequency). These trait changes need not be directly related to the 'state' activity underlying the hallucinations themselves, but influence this activity when it occurs. To this end we investigated cognitive profiles and resting-state functional connectivity couplings associated with $\mathrm{MVH}$ and $\mathrm{CVH}$ temporal phenomenology. In terms of cognitive profile we hypothesized that MVH are associated with visuospatial impairment due to dorsal visual stream involvement, whereas $\mathrm{CVH}$ are associated with visuoperceptual impairment due to ventral-visual stream involvement. In terms of networks, based on ffytche's VH model [14], we hypothesized functional alterations between the brainstem and visual cortices and within the dorsal visual stream to underpin $\mathrm{MVH}$, and functional alterations within the ventral-visual stream to underpin CVH. Given the lack of previous evidence and exploratory nature of our analysis of temporal aspects of hallucination duration and frequency we had no specific hypotheses as to what specific aspects of connectivity would relate to temporal phenomenology.

\section{Materials and methods}

\section{Participants}

We included both DLB and PDD participants consistent with the view that both are part of the LBD spectrum [3, 21]. Thirty-nine LBD patients (21 with DLB and 18 with PDD) with a history of VH (as defined below) were recruited from the Department of Human Neuroscience of Sapienza University Hospital of Rome. Patients were considered trait hallucinators if they had experienced repeated hallucinations during the course of their illness. The majority of these patients had ongoing $\mathrm{VH}$ at the time of recruitment or the preceding months. We set an arbitrary cut off of having hallucinations in the preceding five years and a minimum of two separate hallucination episodes to define the hallucination trait but, in practice, the average time since the last hallucination was 2.6 months for $\mathrm{CVH}$ and 4.8 months for $\mathrm{MVH}$ with a maximum of 36 months (see results). DLB diagnosis was made according to the consensus criteria for probable DLB [22], whereas PDD diagnosis was made according to the Movement Disorder Society Clinical Diagnostic Criteria for Parkinson's Disease [23]. Exclusion criteria for all participants were: MRI contraindications, a previous history of alcohol or substance abuse, significant neurological or psychiatric history, severe ocular diseases (e.g. glaucoma, macular degeneration), epilepsy, other forms of dementia, focal brain lesions on brain imaging, or the presence of other severe or unstable medical illnesses. All participants or their caregivers gave their written informed consent for the study, which was approved by the local ethics committee. 


\section{Clinical and neuropsychiatric assessment}

All patients underwent a neurological examination including the Unified Parkinson's Disease Rating Scale (UPDRS) part III [24] and a structured clinical interview with caregivers to assess the presence of at least two of the core DLB clinical features: parkinsonism (i.e. hypokinesia, rest tremor, postural instability, rigidity), REM sleep behavioral disorders, cognitive fluctuations, and VH. All participants had normal or corrected to normal vision and a gross deficit in color perception was excluded by clinical screening in which patients were asked to indicate a specific color among many different color targets. Current treatment with levodopa, dopamine agonists (DA), antipsychotics, and acetylcholinesterase inhibitors (AchEI), e.g. rivastigmine, was also recorded. Levodopa and DA dosage were converted using the levodopa equivalent scale [25], while antipsychotic drug dosage was converted using the chlorpromazine scale [26]. Neuropsychiatric assessment was performed using the Neuropsychiatric Inventory (NPI) [27, 28].

\section{Visual hallucination assessment}

A modified version of the North-East Visual Hallucinations Interview (NEVHI) [29] was administered to all patients and their caregivers by experienced clinicians/researchers. The NEVHI is a semi-structured interview that assesses in detail the phenomenology of $\mathrm{VH}$ including its temporal aspects and time of last $\mathrm{VH}$ and its emotional, social, and behavioral impact. The modified version included separate sections for simple, presence, illusion, complex, and passage hallucinations and other visual phenomena with questions about their frequency and time duration. The duration question asks "Approximately how long do these experiences usually last?" and the possible answers are "seconds", "minutes", "hours", or "continuous", scored respectively from 1 to 4. The frequency question asks "How often do they usually occur?" and the possible answers are: "less than every few months", "every few months", "every few weeks", "every few days", "every few hours", "every few minutes", "every few seconds", or "continuously", scored respectively from 1 to 8 . The following temporal severity scores were derived:

MVH severity - the sum of duration X frequency for illusions, presence and passage and other (possible score $4-128)$.

MVH duration- the sum of duration for illusions, presence and passage and other (possible score $4-16$ ).

MVH frequency-the sum of frequency for illusions, presence and passage and other (possible score 4-32).

CVH severity-duration X frequency for complex (possible score $1-32$ ).

CVH duration-duration complex (possible score 1-4).
CVH frequency-frequency complex (possible score 1 $-8)$.

Simple hallucinations are considered distinct from $\mathrm{MVH}$ or $\mathrm{CVH}$ [30] and were only reported by three of the participants (see results) so were not included in the scale.

\section{Neuropsychological assessment}

All patients underwent the Mini-Mental State Examination (MMSE) [31] to measure global cognitive decline, as well as the Activities of Daily Living (ADL) and Instrumental Activities of Daily Living (IADL) [32] tests to assess functional impairment. Patients underwent an extensive neuropsychological evaluation including: (1) Rey's Auditory Verbal Learning Test [33], Corsi Block-Tapping Test [34], Digit Span [35], Babcock Story Recall Test [34], and Rey-Osterrieth Complex Figure Test [33] for the memory domain; (2) the Visual Search Test [34] and Trail-Making Test [36] for the attentional domain; (3) the Clock Drawing Test [37] and Copy of the Rey-Osterrieth Complex Figure Test [33] for visuo-constructional skill assessment; (4) Semantic and Phonemic Verbal Fluency Test and Boston Naming Test for language [38]; (5) Raven's Colored Progressive Matrices (RCPM) [39] for abstract reasoning ability and (6) the Frontal Assessment Battery [40] for executive functions. On a different day, patients also underwent the Visual Object and Space Perception battery (VOSP) [41], and the "Length Match Task", "Size Match Task", "Orientation Match Task"; "Position of Gap Match Task" subtests of the Birmingham Object Recognition Battery (BORB) [42] and the Benton lines test [43], to assess early visual processing abilities and complex visuoperceptual functions. Visuoperceptual and visuospatial accuracy indices were computed for each participant using VOSP subtest scores as follows: the visuoperceptual index corresponded with the mean of the proportions of correct answers on the Incomplete Letters, Silhouettes, Object Decision, and Progressive Silhouettes; the visuospatial index corresponded to the mean of the proportions of correct answers on the Dot Counting, Position Discrimination, Number Location and Cube Analysis. Since, a higher score corresponds to a worse performance on the silhouette subtest, while a higher score indicates better performance on all other VOSP subtests, we inverted the silhouette score before computing the index in order to homogenize the correspondence between score and performance. A BORB accuracy index was also computed as the mean of the proportion of correct answers on the administered subtests.

\section{Statistical analysis}

Statistical analyses were performed using SPSS (v. 25). First, spearman correlation coefficients were computed to 
test the association between $\mathrm{MVH}$ and $\mathrm{CVH}$ severity scores and demographics (age and education), concomitant medications (levodopa, DA, AchEI, antipsychotics), and parkinsonism severity (UPDRS). Spearman correlation coefficients were used to test the association between $\mathrm{MVH}$ and $\mathrm{CVH}$ severity, duration, and frequency scores and neuropsychological test scores with significance threshold of $p<0.05$ Bonferroni corrected for 150 multiple comparisons (equivalent to uncorrected $p<0.0003$ ).

\section{Image acquisition and analyses}

MRI scans were collected on a Siemens Magnetom Verio 3-Tesla scanner. Functional T2*-weighted images were collected using a gradient echo sequence to measure the blood oxygen level-dependent (BOLD) contrast over the whole brain. During resting-state fMRI scans the patients were taking their routine medication and asked to lay at rest with eyes closed and not to fall asleep. Head movements were minimized with a mild restraint and cushioning. Functional MRI images were acquired in the interleaved mode for the entire cortex using BOLD contrast imaging (200 fMRI scans, 50 slices, in-plane resolution $=3 \times 3 \mathrm{~mm}$, slice thickness $=3 \mathrm{~mm}$, repetition time $(\mathrm{TR})=3 \mathrm{~s}$, echo time $(\mathrm{TE})=31 \mathrm{~ms})$. Resting-state data were processed using the CONN toolbox for functional connectivity analysis (v. 16a) [44] (http://www.nitrc.org/projects/conn) running on Statistical Parametric Mapping 12 (SPM12) software (http://www. fil.ion.ucl.ac.uk/spm). We used the standard pre-processing pipeline in the CONN toolbox. In brief, after removal of the initial four scans, the functional images were resampled to a voxel size of $2 \times 2 \times 2 \mathrm{~mm}^{3}$, realigned and unwarped; time series were interpolated to correct for slice-timing distortions. Structural images were segmented in gray matter, white matter (WM), and cerebrospinal fluid (CSF) for successive use during removal of temporal confounding factors and normalized to MNI space. ART-based scrubbing [45] for detection of functional outliers was also applied (data showing framewise displacement greater than $0.9 \mathrm{~mm}$ or with a z-normalized signal change over the whole brain greater than 5 were discarded along with the preceding and the following two timepoints). According to the default settings, seed-to-seed connectivity analysis was performed on unsmoothed data aggregated across all voxels within each seed ROI. Temporal confounding factors (i.e. time-courses of WM and CSF BOLD signals, a linear trend, and the six motion parameters derived from the previous realignment procedure) were removed from the BOLD time series of functional data, regressing them out at each voxel. A bandpass filter $(0.008-0.09 \mathrm{~Hz})$ was then applied to resulting residual time series.

In this study a model-based/hypothesis-driven analysis approach was used to test intrinsic functional coupling between seed regions we expected to be involved in $\mathrm{VH}$, based on previous studies and theoretical models (Supplementary Table 1). Thus, we included as seeds early visual areas, i.e. Occipital pole (OP) and Cuneal cortex, regions of the ventral-visual stream, i.e. temporo occipital fusiform cortex (TOFusC) and occipital fusiform gyrus (OFusG), Lingual gyrus (LG), inferior division of lateral occipital cortex (iLOC), temporo occipital part of inferior temporal gyrus (toITG), anterior and posterior division of parahippocampal gyrus (aPaHC, pPaHC), regions of the dorsal visual stream, i.e. superior division of lateral occipital cortex (sLOC), temporo occipital part of middle temporal gyrus (toMTG), regions of the salience network, i.e., anterior and posterior division of supramarginal gyrus (aSMG, pSMG), and regions part of the default mode network, i.e. angular gyrus (AG), posterior division of cingulate gyrus (PC), Precuneus and finally brain stem based on ffytche's hypothesis [14]. Since we were interested in the intrahemispheric connectivity of these nodes, and given that resting-state signal fluctuations in cortical regions tend to be highly positively correlated between homologous regions across the two hemispheres, we analyzed each hemisphere separately, as follows. Using multiple regression models, we performed a seed-to-seed functional connectivity (FC) analysis to disclose possible specific nodes whose functional coupling was associated with the: (i) severity, (ii) duration, and (iii) frequency of MVH (controlling for $\mathrm{CVH}$ and MMSE) and CVH (controlling for MVH and MMSE) for the right and the left hemisphere. Cluster-level inference was adopted to control for family-wise error rate, as implemented in Conn (20.b; CONN toolbox www.nitrc. org/projects/conn, RRID:SCR_009550). In brief, rather than focusing on individual connections between all possible pairs of ROIs, cluster-level inference focuses on groups of nearby or related connections sharing similar effects or results. Here we used default hierarchical clustering method and parametric multivariate statistics (Functional Network Connectivity; [46], with a cluster threshold of $\mathrm{p}<0.05$ cluster-level $\mathrm{p}$-FDR corrected (MVPA omnibus test) and a connection threshold of $p<0.05 \mathrm{p}$-uncorrected.

\section{Results}

Data from 35 out of 39 patients were analyzed, since 2 patients were excluded due to a visual impairment and 2 were excluded from fMRI analysis due to movement artifacts. The final sample included 19 DLB patients and 16 PDD patients. The sample included 23 males $(65.7 \%)$. Mean age was $76.7( \pm 6.5)$ years, mean time from disease onset was $4.3( \pm 2.8)$ years, mean time since last $\mathrm{CVH}$ 
Table 1 Demographics and clinical characteristics of LBD patients (DLB $n=19$, PDD $n=16$ )

\begin{tabular}{ll}
\hline Variable & Mean $( \pm \mathrm{SD})$ \\
\hline Age (years) & $76.7( \pm 6.5)$ \\
Education (years) & $9( \pm 3.9)$ \\
Gender (Male, number and \%) & $23(65.7 \%)$ \\
Time from disease onset (years) & $4.3( \pm 2.8)$ \\
MMSE & $20.7( \pm 5.8)$ \\
UPDRS & $23.3( \pm 11.7)$ \\
NPI total score & $28.9( \pm 16.1)$ \\
NEVHI total score & $11.7( \pm 10.9)$ \\
NEVHI Severity of minor hallucinations & $4.7( \pm 8.2)$ \\
NEVHI Severity of complex hallucinations & $6.9( \pm 4.9)$ \\
NEVHI duration of minor hallucinations & $1.4( \pm 1.8)$ \\
NEVHI duration of complex hallucinations & $3.5( \pm 1.9)$ \\
NEVHI frequency of minor hallucinations & $2.6( \pm 3.02)$ \\
NEVHI frequency of complex hallucinations & $3.5( \pm 1.7)$ \\
Time from last CVH episode (months) & $2.6( \pm 6.8)$ \\
Time from last MVH episode (months) & $4.8( \pm 6.2)$ \\
AcheI (number of patients in therapy and $\%)$ & $19(54.2 \%)$ \\
AcheI dose & $5.4( \pm 4.3)$ \\
Levodopa (number of patients in therapy and $\%)$ & $19( \pm 54.2 \%)$ \\
Levodopa equivalent dose & $364.1( \pm 378.8)$ \\
Antipsychotics (number of patients in therapy and & $14(40 \%)$ \\
$\%)$ & \\
Antipsychotics (Chlorpromazine equivalent dose) & $36( \pm 57.3)$ \\
\hline
\end{tabular}

was $2.6( \pm 6.8)$ months, mean time since last MVH was $4.8( \pm 6.2)$ months and the mean MMSE score was 20.7 $( \pm 5.8)$, indicating a mild global cognitive impairment on average. All the participants were right-handed. Demographic and clinical data are shown in Table 1.

Nine patients had illusions, 9 patients had presence hallucinations, 12 had passage hallucinations, 32 had complex hallucinations ( 3 patients had minor phenomena unassociated with CVH (Fig. 1). Only three patients reported simple visual hallucinations. None of the patients experienced VH during resting-state fMRI scanning or reported falling asleep.

No significant correlations were found between MVH and $\mathrm{CVH}$ severity scores, and age, education, time from disease onset, concomitant medications, or UPDRS score.

\section{Neuropsychological results}

No significant correlations were found between cognitive test scores and MVH severity (the mean test scores are shown in Supplementary Table 2 and Spearman's correlation coefficients are reported in Table 2). We explored the possibility that the lack of significant correlation might be a statistical artifact related to 0 values (i.e. patients who did

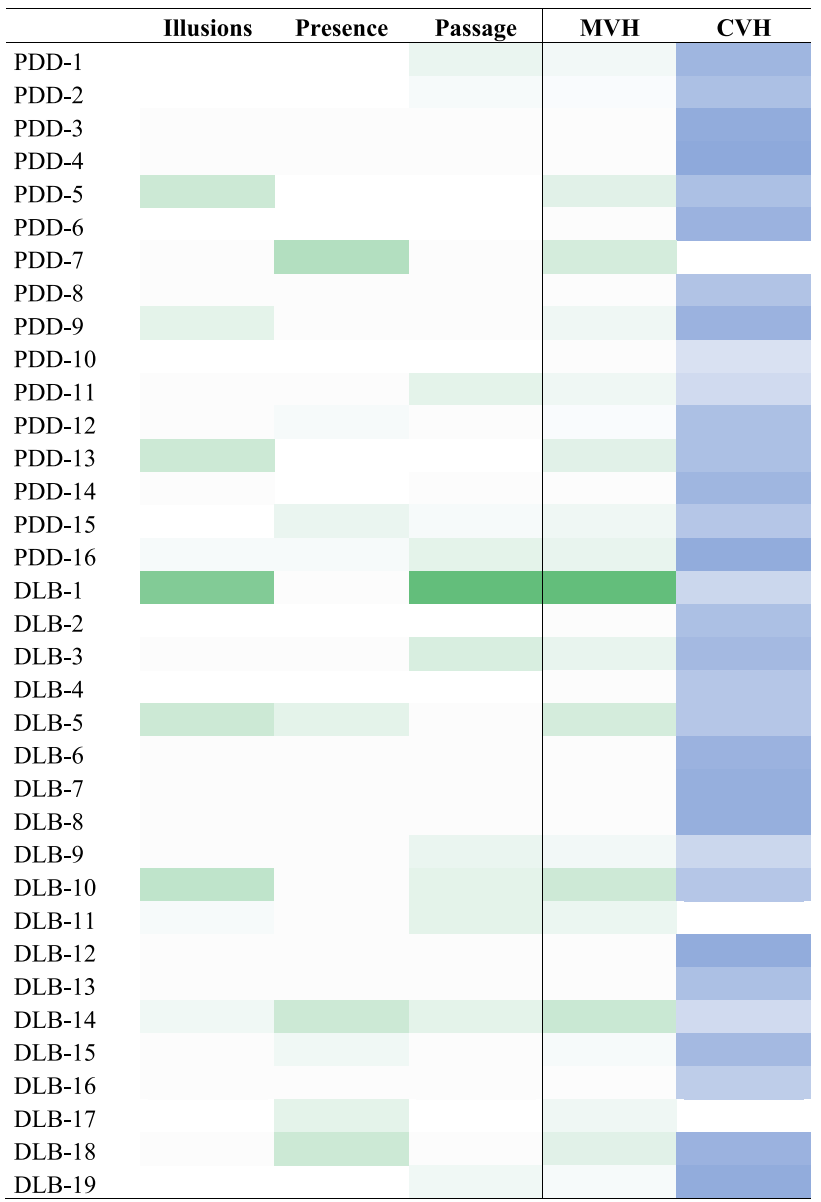

Fig. 1 Colored patches of visual phenomena in LBD patients. Minor visual hallucinations, $M V H$ light green-to-dark green; complex visual hallucinations, $\mathrm{CVH}$ light blue-to-dark blue represent the severity of symptoms reported by each patient for each phenomenon

not report MVH). We therefore repeated the analysis without participants with 0 values and also looked for a difference in cognitive score between patients with and without MVH. Neither analysis suggested an association between MVH and cognitive test score, other than at trend significance for TMT-A and TMT-B (see Supplementary Table 3 and Supplementary Table 4). In contrast, a negative correlation was detected between CVH severity and MMSE $(r=-0.568$, $\left.\mathrm{p}_{\text {corr }}<0.05\right)$, VOSP visuoperceptual index $(r=-0.625$, $\left.p_{\text {corr }}<0.05\right)$, RCPM $\left(r=-0.581, p_{\text {corr }}<0.05\right)$ and visual search scores $\left(r=-0.622, p_{\text {corr }}<0.05\right)$ at trend significance for VOSP spatial index ( $r=-0.562$, uncorrected $p=0.002)$. $\mathrm{CVH}$ duration was negatively associated with RCPM score $\left(r=-0.577, p_{\text {corr }}<0.05\right)$ and at trend significance for VOSP visuoperceptual index $(r=-0.535$, uncorrected $p=0.003)$, VOSP spatial index $(r=-0.559$, uncorrected $p=0.002)$, visual search scores $(r=-0.496$, uncorrected $p=0.003)$ and MMSE $(r=-0.471$, uncorrected $p=0.004)$. 
Table 2 Spearman correlation coefficients (r) between Minor (MVH) and complex visual hallucinations (CVH) scores and neuropsychological test scores and significance $(p)$

\begin{tabular}{|c|c|c|c|c|c|c|c|}
\hline Test & Value & MVH severity & CVH severity & MVH duration & CVH duration & MVH frequency & $\mathrm{CVH}$ frequency \\
\hline \multirow[t]{2}{*}{ MMSE } & $r$ & 0.045 & $-0.568^{*}$ & 0.069 & -0.471 & -0.010 & -0.450 \\
\hline & $p$ & 0.797 & 0.000 & 0.694 & 0.004 & 0.957 & 0.007 \\
\hline \multirow[t]{2}{*}{ Benton } & $r$ & -0.073 & -0.357 & -0.017 & -0.314 & -0.111 & -0.285 \\
\hline & $p$ & 0.706 & 0.057 & 0.932 & 0.097 & 0.565 & 0.135 \\
\hline \multirow[t]{2}{*}{ VOSP visuo } & $r$ & -0.070 & $-0.625^{*}$ & -0.020 & -0.535 & -0.084 & -0.497 \\
\hline & $p$ & 0.717 & 0.000 & 0.917 & 0.003 & 0.663 & 0.006 \\
\hline \multirow[t]{2}{*}{ VOSP spatial } & $r$ & -0.121 & -0.562 & -0.078 & -0.559 & -0.127 & -0.406 \\
\hline & $p$ & 0.530 & 0.002 & 0.687 & 0.002 & 0.513 & 0.029 \\
\hline \multirow[t]{2}{*}{$B O R B$} & $r$ & -0.083 & -0.412 & -0.077 & -0.379 & -0.094 & -0.364 \\
\hline & $p$ & 0.682 & 0.033 & 0.704 & 0.051 & 0.641 & 0.062 \\
\hline \multirow[t]{2}{*}{$D S$} & $r$ & -0.135 & -0.402 & -0.107 & -0.412 & -0.094 & -0.208 \\
\hline & $p$ & 0.455 & 0.020 & 0.555 & 0.017 & 0.601 & 0.246 \\
\hline \multirow[t]{2}{*}{$C B T$} & $r$ & 0.000 & -0.363 & 0.040 & -0.316 & 0.009 & -0.221 \\
\hline & $p$ & 0.998 & 0.038 & 0.825 & 0.073 & 0.961 & 0.216 \\
\hline \multirow[t]{2}{*}{$R A V L I R$} & $r$ & 0.047 & -0.238 & 0.067 & -0.286 & 0.046 & -0.108 \\
\hline & $p$ & 0.796 & 0.183 & 0.711 & 0.107 & 0.800 & 0.549 \\
\hline \multirow[t]{2}{*}{$R A V L D R$} & $r$ & 0.197 & -0.317 & 0.240 & -0.361 & 0.226 & -0.221 \\
\hline & $p$ & 0.271 & 0.072 & 0.178 & 0.039 & 0.205 & 0.217 \\
\hline \multirow[t]{2}{*}{ Babcock IR } & $r$ & 0.089 & -0.114 & 0.128 & -0.121 & 0.030 & -0.115 \\
\hline & $p$ & 0.629 & 0.536 & 0.484 & 0.510 & 0.870 & 0.530 \\
\hline \multirow[t]{2}{*}{ Babcock DR } & $r$ & -0.085 & -0.235 & -0.043 & -0.194 & -0.076 & -0.198 \\
\hline & $p$ & 0.645 & 0.196 & 0.815 & 0.287 & 0.681 & 0.277 \\
\hline \multirow[t]{2}{*}{ RCFT IR } & $r$ & 0.185 & -0.005 & 0.235 & -0.013 & 0.158 & -0.035 \\
\hline & $p$ & 0.327 & 0.979 & 0.211 & 0.945 & 0.405 & 0.856 \\
\hline \multirow[t]{2}{*}{$R C F T D R$} & $r$ & 0.261 & -0.053 & 0.311 & -0.244 & 0.256 & 0.131 \\
\hline & $p$ & 0.164 & 0.781 & 0.094 & 0.195 & 0.171 & 0.490 \\
\hline \multirow[t]{2}{*}{$T M T-A$} & $r$ & -0.224 & 0.180 & -0.237 & 0.211 & --0.155 & 0.059 \\
\hline & $p$ & 0.218 & 0.324 & 0.192 & 0.247 & 0.397 & 0.750 \\
\hline \multirow[t]{2}{*}{$T M T-B$} & $r$ & -0.376 & 0.059 & -0.363 & 0.048 & -0.355 & 0.044 \\
\hline & $p$ & 0.064 & 0.780 & 0.075 & 0.818 & 0.081 & 0.835 \\
\hline \multirow[t]{2}{*}{$V S$} & $r$ & -0.177 & $-0.622 *$ & -0.155 & -0.496 & -0.210 & -0.558 \\
\hline & $p$ & 0.323 & 0.000 & 0.390 & 0.003 & 0.240 & 0.001 \\
\hline \multirow[t]{2}{*}{$P V F$} & $r$ & -0.052 & -0.350 & -0.039 & -0.288 & -0.038 & -0.244 \\
\hline & $p$ & 0.772 & 0.046 & 0.831 & 0.104 & 0.835 & 0.171 \\
\hline \multirow[t]{2}{*}{$S V F$} & $r$ & -0.202 & -0.461 & -0.209 & -0.363 & -0.279 & -0.302 \\
\hline & $p$ & 0.269 & 0.008 & 0.252 & 0.041 & 0.123 & 0.093 \\
\hline \multirow[t]{2}{*}{$B N T$} & $r$ & 0.094 & -0.529 & 0.137 & -0.496 & 0.065 & -0.368 \\
\hline & $p$ & 0.607 & 0.002 & 0.453 & 0.004 & 0.723 & 0.039 \\
\hline \multirow[t]{2}{*}{$C D T F D$} & $r$ & -0.049 & -0.508 & 0.015 & -0.371 & -0.111 & -0.420 \\
\hline & $p$ & 0.816 & 0.009 & 0.942 & 0.068 & 0.596 & 0.037 \\
\hline$C D T E D$ & $r$ & 0.074 & -0.419 & 0.122 & -0.267 & 0.034 & -0.351 \\
\hline & $p$ & 0.725 & 0.037 & 0.562 & 0.197 & 0.870 & 0.086 \\
\hline$C D T P D$ & $r$ & -0.151 & -0.421 & -0.067 & -0.309 & -0.155 & -0.308 \\
\hline & $p$ & 0.463 & 0.032 & 0.744 & 0.124 & 0.450 & 0.126 \\
\hline RCFT copy & $r$ & 0.086 & -0.210 & 0.149 & -0.233 & 0.032 & -0.151 \\
\hline & $p$ & 0.647 & 0.257 & 0.422 & 0.207 & 0.864 & 0.417 \\
\hline$R C P M$ & $r$ & -0.172 & $-0.581^{*}$ & -0.131 & $-0.577^{*}$ & -0.162 & -0.395 \\
\hline & $p$ & 0.340 & 0.000 & 0.466 & 0.000 & 0.367 & 0.023 \\
\hline$F A B$ & $r$ & -0.116 & -0.330 & -0.100 & -0.260 & -0.154 & -0.290 \\
\hline & $p$ & 0.527 & 0.065 & 0.588 & 0.150 & 0.399 & 0.107 \\
\hline
\end{tabular}

Significant correlation coefficients with CVH that survived the Bonferroni's correction for multiple comparison are marked with an asterisk Notes: $I R$ : immediate recall, $D R$ delayed recall, $R A V L T$ Rey's auditory verbal learning test, $D S$ Digit span, $C B T$ Corsi block tapping test, $R C F T$ 
Table 2 (continued)

Rey-Osterrieth complex figure test, $V S$ visual search test, TMT-A trail making test part A, TMT-B trail-making test part B, $P V F$ phonemic verbal fluency, $S V F$ semantic verbal fluency, $B N T$ boston naming test, $C D T$ clock drawing test, $F D$ free drawing condition, $P D$ pre-drawn condition, $E D$ examiner-drawn condition, $R C P M$. Raven's colored progressive matrices; $F A B$ frontal assessment battery

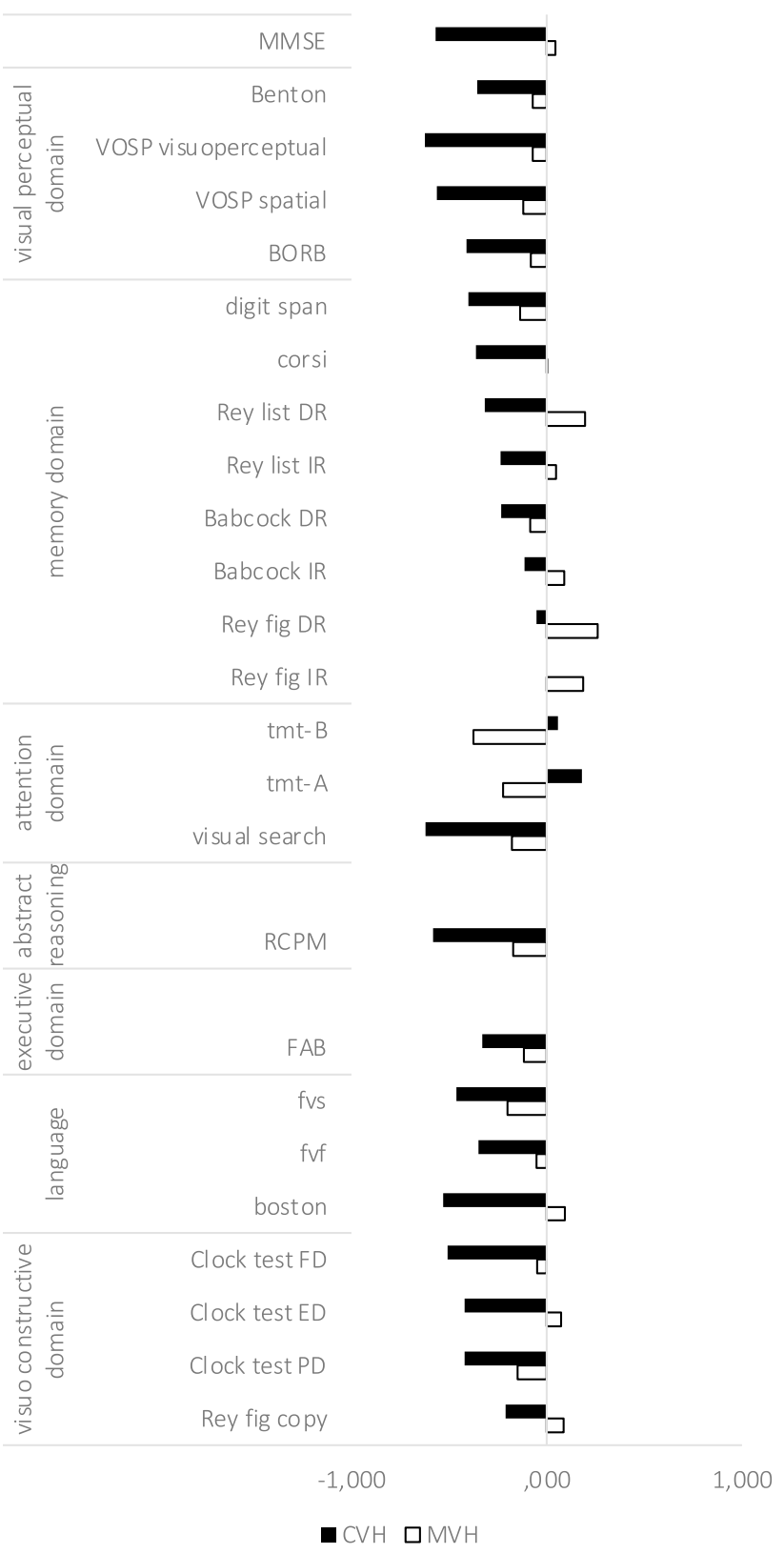

Fig. 2 Bars summarize Spearman's coefficients between minor $(\mathrm{MVH})$ and complex $(\mathrm{CVH})$ severity obtained from NEVHI and neuropsychological data. Abbreviations: $I R$ immediate recall, $D R$ delayed recall, TMT-A Trail-Making Test part A, TMT-B Trail-Making Test part B, RCPM Raven's colored progressive matrices, FAB Frontal Assessment Battery, Clock test FD free drawing condition, $P D$ predrawn condition, $E D$ examiner-drawn condition, $P V F$ Phonemic Verbal Fluency, SVF Semantic Verbal Fluency
The correlation trends between complex and minor hallucinations and neuropsychological domains are shown in Fig. 2. Spearman's correlation coefficients are reported in Table 2 along with significance.

\section{Neuroimaging results}

\section{MVH seed-to-seed results}

Figure 3 shows the network of related ROIs sharing similar functional metrics defined using the hierarchical clustering procedure, and, in this network, the regions showing suprathreshold functional couplings associated with minor visual phenomena. Specifically, the multiple regression models revealed that seed-to-seed FC between brain stem and OP and iLOC was negatively associated with MVH severity in the left hemishere. Also, FC between posterior parahippocampal gyrus and iLOC and between anterior parahippocampal gyrus and OP were negatively associated with MVH severity in the left hemisphere. No significant effects were detected in the right hemisphere. Moreover, MVH duration and frequency were not associated with FC alterations neither in the right or left hemisphere. Statistics are reported in Table 3.

\section{CVH seed-to-seed results}

Figure 4 shows the networks of related ROIs sharing similar functional metrics defined using the hierarchical clustering procedure, and the regions showing suprathreshold functional couplings associated with $\mathrm{CVH}$ duration and frequency. Thus, CVH severity was not associated with FC alterations, neither in the right or left hemisphere. However, FC between pSMG and LG, TOFus and OP was positively associated with $\mathrm{CVH}$ duration in right hemisphere. Also, FC between Precuneus and right aSMG and pSMG was negatively associated with $\mathrm{CVH}$ frequency, in the right hemisphere; FC between right PC and right pSMG and between right $\mathrm{AG}$ and right $\mathrm{pSMG}$ was negatively associated with $\mathrm{CVH}$ frequency in the right hemisphere, as well. Finally, FC between AG and SMG was negatively associated with CVH frequency in the left hemisphere. No significant associations with CVH duration were found in the left hemisphere. Statistics are reported in Table 4. 


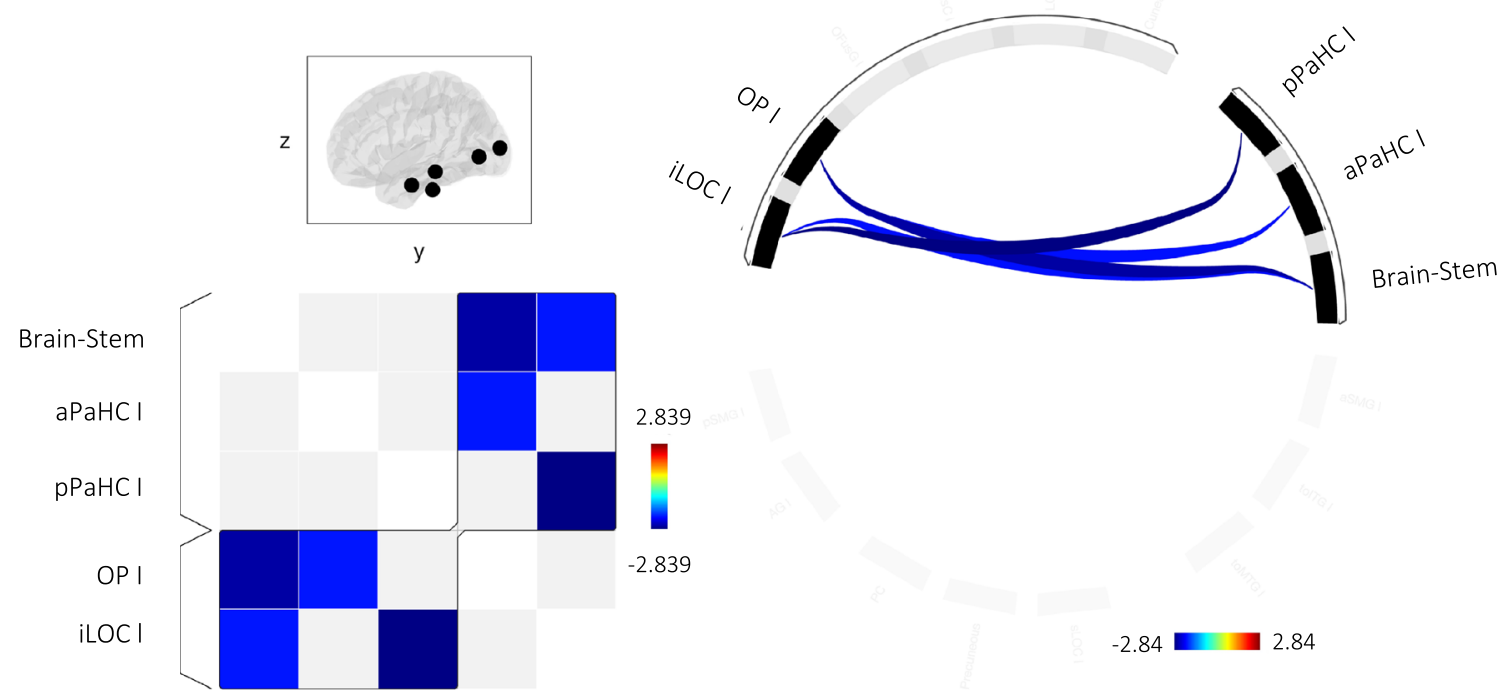

Fig. 3 Network of minor visual phenomena. Groups of related ROIs has been defined using hierarchical clustering procedure. Significant connections were identified using a cluster threshold of $p<0.05$ cluster-level $p$-FDR corrected (MVPA omnibus test) and a connection threshold of $p<0.05$ p-uncorrected. Regions showing suprath- reshold functional couplings associated with minor visual phenomena (i.e. severity) are depicted in light blue-to blue patches (on the left panel) and line (on the right panel). Notes: $l$ left, $a P a H C$ anterior parahippocampal cortex, $\mathrm{PPaHC}$ posterior parahippocampal cortex, $O P$ occipital pole, $i L O C$ inferior lateral occipital complex

Table 3 Significant associations between minor visual hallucinations and regional functional couplings

\begin{tabular}{|c|c|c|c|c|c|}
\hline & Seed & & $\mathrm{T}_{(31)}$ & p-unc & $\mathrm{p}-\mathrm{FDR}$ \\
\hline \multicolumn{6}{|c|}{ Right hemisphere } \\
\hline \multicolumn{6}{|l|}{ Severity } \\
\hline & & No significant suprathreshold cluster & & & \\
\hline \multicolumn{6}{|l|}{ Duration } \\
\hline & & No significant suprathreshold cluster & & & \\
\hline \multicolumn{6}{|c|}{ Frequency } \\
\hline & & No significant suprathreshold cluster & & & \\
\hline \multicolumn{6}{|c|}{ Left hemisphere } \\
\hline \multicolumn{6}{|l|}{ Severity } \\
\hline & & $\mathrm{F}(2,30)=8.76$ & & 0.001 & 0.015 \\
\hline & Brain stem & Occipital pole 1 & -2.65 & 0.012 & \\
\hline & Posterior parahippocampal gyrus 1 & Inferior division lateral occipital cortex 1 & -2.84 & 0.007 & \\
\hline & Brain stem & Inferior division lateral occipital cortex 1 & -2.05 & 0.049 & \\
\hline & Anterior parahippocampal gyrus 1 & Occipital pole 1 & -2.04 & 0.049 & \\
\hline \multicolumn{6}{|l|}{ Duration } \\
\hline & & No significant suprathreshold cluster & & & \\
\hline \multicolumn{6}{|l|}{ Severity } \\
\hline & & No significant suprathreshold cluster & & & \\
\hline
\end{tabular}

Notes on region labels: $1=$ left hemisphere

\section{Discussion}

The present study investigated alterations in cognitive functioning and resting-state functional couplings in patients with LBD susceptible to VH (hallucination trait). Neuropsychological and functional coupling alterations were identified when patients were not hallucinating that correlated with temporal aspects of the $\mathrm{VH}$ experience itself (hallucination state), suggesting $\mathrm{VH}$ trait alterations 
Fig. 4 Network of complex visual hallucinations. Groups of related ROIs has been defined using hierarchical clustering procedure. Significant connections were identified using a cluster threshold of $p<0.05$ cluster-level p-FDR corrected (MVPA omnibus test) and a connection threshold of $p<0.05$ p-uncorrected. Regions showing suprathreshold functional couplings associated with complex visual hallucinations are depicted in light blue-to blue patches (on the left) and line (on the right) when a negative relation has been found; otherwise, positive association with complex visual hallucinations are depicted in yellow-to-red patches and lines. Panels display the resting-state functional couplings whose strengths showed a significant association with duration of complex visual hallucinations in the right hemisphere (A) and that with frequency in the right (B) and left hemisphere (C). Notes: $l$ left, $r$ right, $O P$ occipital pole, $P C$ posterior cingulate cortex, $A G$ angular gyrus, $p S M G$ posterior supramarginal gyrus, $a S M G$ anterior supramarginal gyrus, $L G$ lingual gyrus, TOFusC temporoccipital fusiform cortex
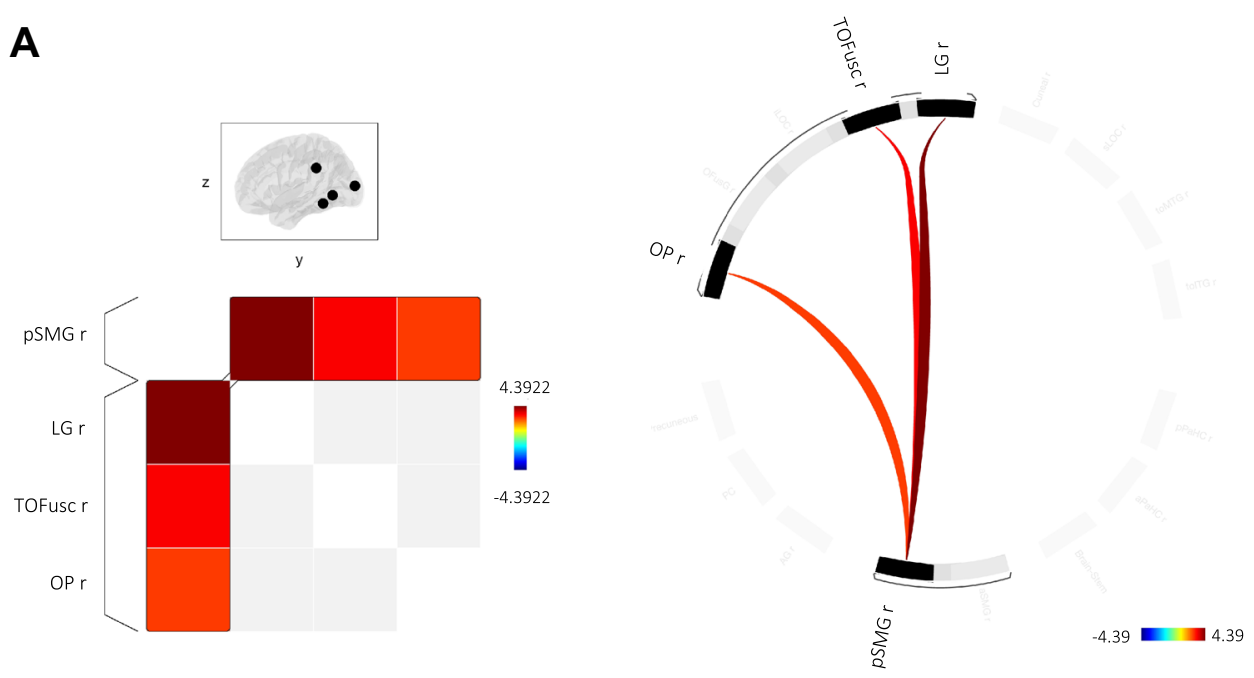

B
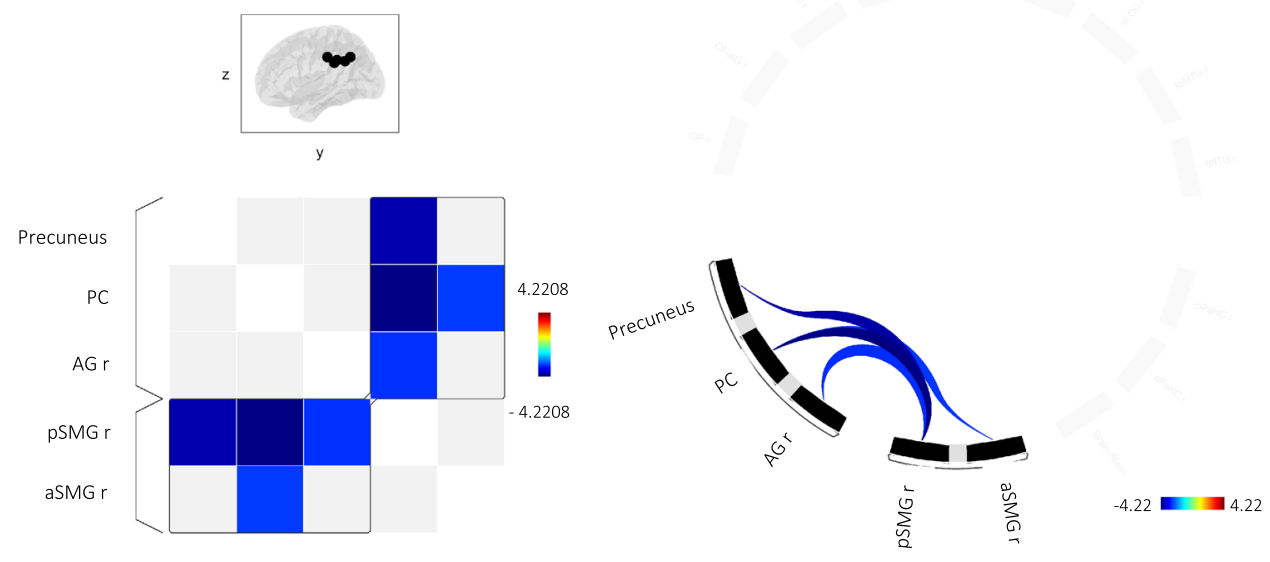

C
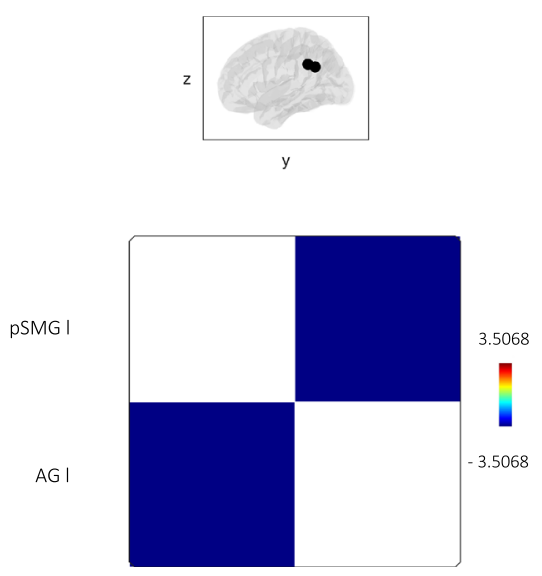

influenced VH state activity. By examining minor and complex VH separately, we tested the hypothesis that temporal aspects of the two classes of phenomena are underpinned by distinct neuropsychological profiles and functional networks. In support of this hypothesis, we found $\mathrm{MVH}$ were not associated with cognitive impairment while CVH were associated with abstract reasoning, visuoperceptual, and attentional alterations and that 
Table 4 Significant associations between duration and frequency of complex visual hallucinations and regional functional couplings

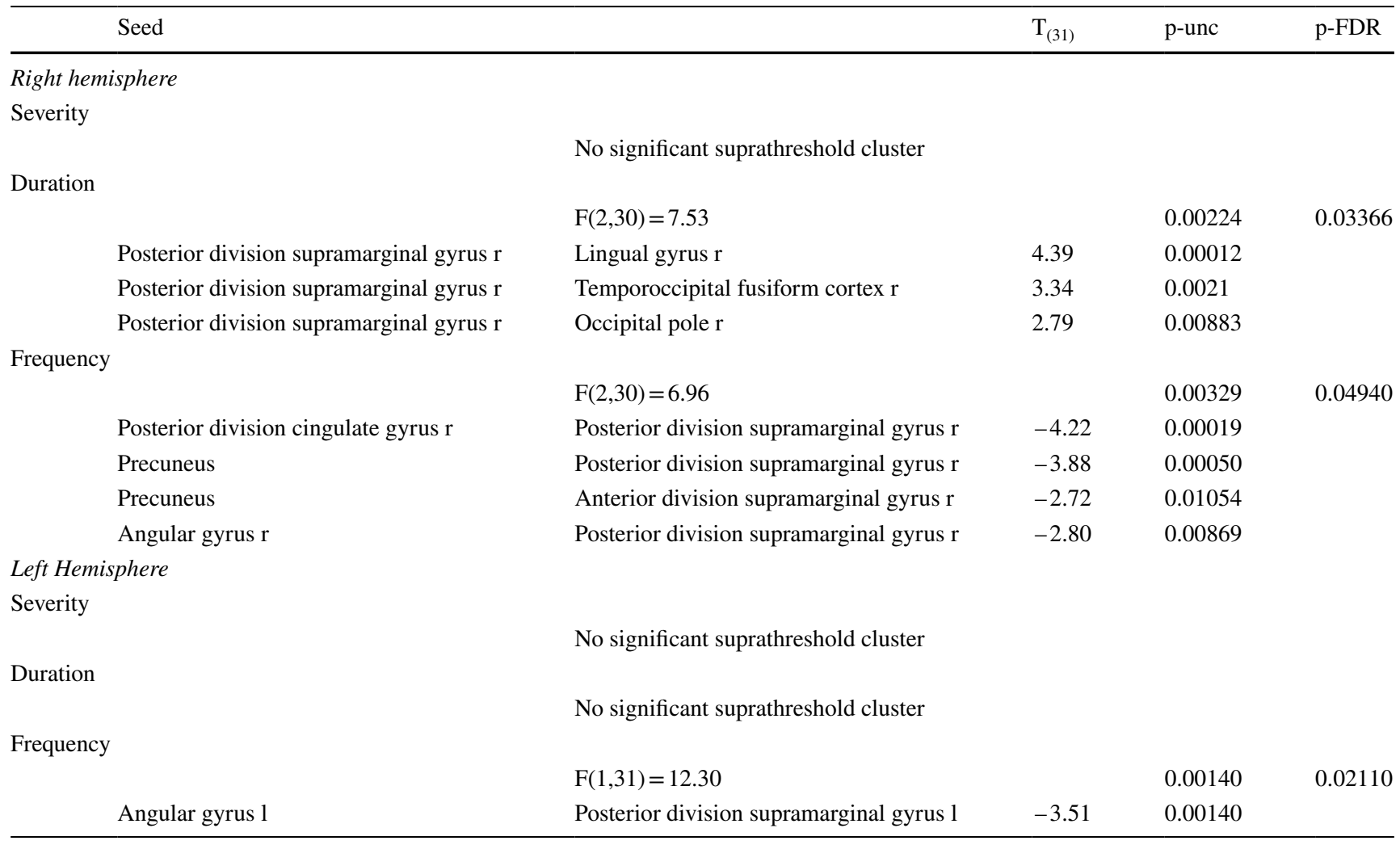

Notes on region labels: 1 left hemisphere, $r$ right hemisphere

functional alterations across visual and related networks differed for MVH and CVH. For easiness of exposition the discussion will be divided into subheadings, concerning the cognitive profile we found to be associated with temporal aspects of $\mathrm{VH}$ in LBD, and functional networks associated with MVH and CVH.

\section{Cognitive profile associated with VH in LBD}

When cognitive score correlations were analyzed separately for $\mathrm{MVH}$ and $\mathrm{CVH}$, no significant associations were found between impairment in any cognitive domain and MVH. These findings suggest that MVH are not associated with impairments in cognitive and perceptual processes measured by the wide-ranging tests used in this study. It may be an as yet unspecified visual dysfunction produces the "erroneous" perceptions of MVH without cognitive impairment as assessed by the tests employed. We do not think the lack of an association between $\mathrm{MVH}$ and cognitive measures reflects the bias related to zero values as confirmed by the correlations performed excluding participants without MVH (see Supplementary Table 3). This result is consistent with findings of the only previous study that explored the neuropsychological profile associated with $\mathrm{MVH}$, reporting no relation between MVH and cognitive impairments [47]. In contrast CVH were associated with global cognitive impairment and deficits in visuoperceptual processing, visual attention, and abstract reasoning related to visual patterns and textures. These results are consistent with the hypothesis that impairment in visuoperceptual function related to the ventral-visual stream, "what pathway" involved in object recognition [5] is a prerequisite for complex hallucinations. The overall temporal severity of $\mathrm{CVH}$ was related to impairments in visual perception coupled with impaired reality monitoring through attentional, reasoning and global cognition deficits. Of note these associations seemed to be primarily driven by the duration measure (correlation with non-verbal reasoning abilities [Raven's Colored Progressive Matrices, a test in which subjects are presented sequences of visual patterns and textures and asked to choose the next in the sequence] and, at trend, global cognition) and not the frequency measure (see below for discussion of duration versus frequency). For $\mathrm{CVH}$, the results support the validity of the neuropsychological Perceptual and Attention model [5] which states that impairment in visuoperceptual processing may lead to a failure in the bottom-up process, which cannot be compensated by an effective top-down mechanism due to cognitive impairment.

Our results are in line with previous studies that have reported an association between VH in LBD and 
impairments in visuoperceptual function [48, 49], visual recognition [50], visual attention $[11,12]$, attention $[9,10]$. Previous studies also found an impairment in executive functions to be associated with $\mathrm{VH}[12,47,51-53]$. In this study we did not found this association, although we found a correlation between CVH severity and RCPM, that may be also considered a measure of executive function [54-57]. However, previous studies did not investigate possible associations between neuropsychological functions and $\mathrm{VH}$ content or the temporal phenomenology of duration or frequency, which might account for the difference in findings.

Overall our cognitive findings are consistent with the hypothesis we set out to test that the profile of deficits for MVH is distinct from that of CVH. The associations found between temporal aspects of $\mathrm{CVH}$ phenomenology and specific cognitive functions in trait hallucinators suggest these functions influence activity underlying the hallucinations state for CVH. Conversely, the lack of associations found for MVH suggest the same functions do not influence activity underlying the hallucination state for MVH.

\section{Minor hallucinations and FC}

We found FC alterations in trait hallucinators associated with temporal aspects of $\mathrm{MVH}$, the implication being that these network changes influenced activity underlying the MVH state. MVH severity was associated in the left hemisphere with reduced FC between the primary visual cortex and parahippocampal regions as well as with a decreased FC between parahippocampal regions and inferior lateral occipital cortex part of the visual stream. Moreover, we found that a decreased FC between the brain stem and primary visual area and the inferior lateral occipital cortex.

The MVH scale is a composite of illusion, passage and presence subscales and it is likely different nodes are responsible to different phenomenological aspects of MVH content.

The FC alteration with the lateral occipital cortex, a region specifically involved in the object recognition, i.e. iLOC [58], may account for illusions, whereas the altered FC with parahippocampal region may account for passage interpreting this result in light of the parahippocampal role in visuospatial processing. It is known that parahippocampus specifically responds to place and building recognition (i.e. parahippocampal place area), however additional hypotheses have been proposed for its role [59]. Levi et al. proposed that the ventral stream is organized along a center-periphery gradient and that parahippocampus might process objects that are typically in the periphery of the visual field [60].

Also, the parahippacampal region is in close proximity to the newly described area prostriata that specifically processes information from the periphery of the visual field and shows a preferential response for fast motion visual stimuli [61].

An altered connectivity with this region may account for the passage hallucination that typically occur as unformed figures passing fast in the periphery of the eye field. Finally, the decreased connectivity between brainstem and primary visual area and object recognition area supports ffytche's hypothesis that alteration of the subcortical control on visuoperceptual process contributes to minor phenomena [14]. Conversely, we did not find alterations involving dorsal visual stream areas. It is possible that these alterations occur during the hallucination state, whereas alterations in spatial recognition process represent the context (trait) within which minor phenomena, such as illusions, passage and presence hallucinations, occur.

No associations were found between FC and MVH duration and frequency considered separately. The reasons for this are unclear but these phenomena usually occur earlier than CVH during the LBD disease course [14] and are more transient and less temporally defined. The questionnaire measures might not be sensitive enough to show associations with duration or frequency when considered separately but are able to do so when combined in the temporal severity measure.

FC connectivity alterations associated with MVH were found just in the left hemisphere. The reason for this is unclear and may relate to the individual symptom components of the MVH. It might be, for example, dysfunction in left hemisphere networks with a relative specialization for processing local features results in certain types of illusion, contrasting with right hemisphere networks specialized for processing global features [62]. The area prostriata has a left hemisphere bias which may relate to the passage hallucination component of the MVH scale.

Overall our results are consistent with previous structural MRI studies that have shown cortical atrophy in PD patients with minor VH in regions of the visual stream and subcortical atrophy of the superior colliculus [63] and peduncolopontine nucleus, which may affect cholinergic transmission from the brainstem to the cortex [64]. Our results are also consistent with previous functional studies of MVH in PD that reported diffuse hypometabolism in upper brainstem and posterior cortical regions and distributed network alterations within visual associative cortex $[65,66]$.The pattern of FC alterations associated with MVH support ffytche's hypothesis that MVH are underpinned by altered activation from the brainstem to visual areas [14].

\section{Complex hallucinations and FC}

We found FC alterations in trait hallucinators associated with temporal aspects of $\mathrm{CVH}$, the implication being that 
these network changes influenced activity underlying the CVH state.

$\mathrm{CVH}$ duration was associated in the right hemisphere with increased FC between primary visual area, fusiform cortex and the lingual gyrus in the ventral-visual stream and the supramarginal gyrus, part of the SN. These findings suggest that a possible mechanism underlying $\mathrm{CVH}$ is dysregulation among the ventral-visual stream areas and salience network with VH resulting from increased salience attributed to the internally-generated perceptual contents. Shine's model also interpreted the increased engagement of the VAN as a condition of greater salience attributed to the stimuli [6]. Functional connectivity alterations associated with $\mathrm{CVH}$ duration were found just in right hemisphere. This result can be interpreted considering the ventral attention network contribution to $\mathrm{CVH}$. Indeed, VAN has a right hemisphere predominance and its tracts are more represented in the right hemisphere [67]. A previous neuroimaging study investigating VH in DLB patients found that microstructural and functional alterations involving attention networks in the right hemisphere contribute to $\mathrm{VH}$, sustaining that $\mathrm{VH}$ are associated with an abnormal functioning of the VAN in the right hemisphere [68]. Overall these results suggest that altered bottom-up processes related to areas in VAN and SN are implicated for $\mathrm{CVH}$ occurrence.

Conversely, frequency-related $\mathrm{FC}$ revealed that alterations in the DMN and $\mathrm{SN}$ are linked to $\mathrm{CVH}$ in both hemispheres. The angular gyrus and posterior cingulate gyrus are both part of the DMN and were found to be less connected with the supramarginal gyrus of the SN. The role of the angular gyrus in the DMN seems to be linked to the manipulation of conceptual knowledge and mental representations when the mind wanders at rest [69]. Our results suggest that the reduced salience devoted to these internal thoughts is associated with CVH frequency. Contrary to previous studies [8, 16, 17], and to Shine's Attention and Control model, we found a decreased FC in the DMN and SN. A possible explanation is that the Attention and Control model has been tested by means of a paradigm based on ambiguous perceptual stimuli more related to misperception illusions than $\mathrm{CVH}$, so may be more relevant to $\mathrm{MVH}$ than $\mathrm{CVH}$. Also, our study relates specifically to temporal aspects of $\mathrm{VH}$ rather than susceptibly to $\mathrm{VH}$ in general as tested in the Shine paradigm. Our results are in line with findings from previous functional studies reporting altered recruitment of the ventral-visual stream and a large-scale multi-network derangement associated with VH in DLB [70], as well as diffuse desynchronization of the lingual gyrus [71], consistent with posterior/occipital changes typical of DLB [72-74].

Overall the FC results are consistent with the neuropsychological findings of impairments in visuoperceptual abilities, visual attention and visual abstract reasoning associated with CVH. Thus, FC alterations involving ventral-visual stream may explain the visuoperceptual alterations and the decreased FC between DMN and SN may be linked to the alterations in attention and reasoning abilities (linked to the angular gyrus) associated with $\mathrm{CVH}$.

For $\mathrm{CVH}$, duration and frequency considered individually were associated with FC but not the combined severity measure, the opposite of what was found for MVH. This might be explained by differences in the construction of the two scales. CVH relates to just one type of complex phenomenon reported by the patient as the most severe while the MVH severity score is the sum of each individual minor phenomenon severity and captures the range of minor phenomena in addition to temporal aspects.

\section{Network connectivity and hallucination duration and frequency}

This is the first study to explore temporal aspects of hallucinations and we had no specific a priori hypotheses to test. In terms of the hodotopic framework, one might anticipate the duration of hallucinations (lasting seconds, minutes, hours etc.) to be related to persistent, sustained activity in the network underlying the symptoms. One possibility is that this type of driving activity is a consequence of hyperconnection/increased coupling in the network, as reflected in a positive correlation with duration, for example between the SN and ventral-visual stream for CVH. In contrast, the frequency of hallucinations (occurring every month, week, day etc.) might be related to instability within the network either as the result of disconnection / reduced coupling as reflected in a negative correlation with frequency, for example between DMN and SN for CVH. Although speculative, these observations suggest duration and frequency may relate to different aspects of network dysfunction and provide new hypotheses to test in future studies.

\section{General discussion}

The present findings shed light on the mechanisms underpinning VH in LBD. VH may be due to functional alterations in visual networks, with $\mathrm{VH}$ phenomenology being defined by the specific brain regions and networks involved. The involvement of specific areas may reflect a link with the neuropathological progression of LBD spread or involvement of specific neurotransmitter systems in the disease process. Visual network involvement may represent the "primum movens" but it is not sufficient for VH onset or temporal phenomenological aspects such as their duration and frequency. These aspects of phenomenology seem to depend on the interaction between visual networks and those of the DMN and SN and alterations of FC that lead to changes on the balance of sustained and unstable, 'spontaneous' or 'autonomous' activity in networks. 


\section{Clinical applications and future directions}

Investigating $\mathrm{VH}$ according to their phenomenology both in terms of content and temporal characteristics, helps clarify the mechanisms underpinning $\mathrm{VH}$, detecting the areas, networks and network dysfunctions involved. This approach may have significant translational potential in clinical practice. Differences in phenomenology may translate to differences in the underlying neurotransmitter systems involved with implications for treatment [75]. For example, CVH frequent in DLB, may be related to cholinergic dysfunctions from ascending systems, mainly affecting limbic regions and the ventral-visual stream [76, 77]. MVH may be instead considered part of a syndrome also including metamorphopsia typically occurring in conditions linked to the serotonergic system such as hallucinogen persisting perception disorder [78], MDMA [79] and 5-HT2 antagonism [80]. Understanding the neurotransmitter signature of the hallucinations in LBD according to content could help develop effective treatments for specific VH subtypes. Another implication of the phenomenological differences identified is that duration and frequency of $\mathrm{VH}$ may be associated with distinct functional alterations, suggesting different types of treatment may be needed to target different aspects of temporal phenomenology.

\section{Caveats and Limitations}

A limitation of the present study is the indirect nature of the inferences drawn from trait to state hallucinations. While it seems reasonable to infer that associations between trait changes and state phenomenology imply a shared mechanism for both, without a better understanding of the activity occurring at the time of $\mathrm{MVH}$ and $\mathrm{CVH}$ in LBD, we cannot be certain that networks exhibiting FC alterations in trait hallucinators or the cognitive domains implicated are directly related to the hallucination state. In our cohort we did not investigate the role of the retina in $\mathrm{VH}$ occurrence. Since LB retinal burden could also contribute to $\mathrm{VH}$, future studies will clarify how retinal alterations are associated with functional network changes in LBD. Moreover, in this study the patients were not assessed for visual contrast sensitivity, although they were screened for color discrimination deficits.

Another limitation is that we cannot definitively establish the role of specific $\mathrm{MVH}$ and $\mathrm{CVH}$ content (faces or figures for example). Also it was not possible investigate the FC alterations associated with each MVH. Future studies that include LBD patients experiencing only one type of phenomenon could help disentangle this issue. Future studies should also investigate and compare VH with specific phenomenology in different neurodegenerative disorders, also analyzing PD, PDD and DLB separately, in order to clarify whether the findings are generally applicable to neurodegenerative disease or specific to Lewy body dementias.

\section{Conclusions}

The findings of the present study suggest that VH phenomenology, broadly defined including content and temporal aspects of $\mathrm{VH}$, is an important consideration for studies of VH mechanism. Exploring VH from a phenomenological point of view helps identify different brain regions, networks and network dysfunctions involved. In his phenomenologie de la perception (1945), Merlau-Ponty claimed that it is not possible to differentiate the experiencing subject from the experience itself [81]. Restating this in neuroscientific terms it may be that what we perceive or misperceive (i.e. a $\mathrm{VH}$ ) is closely linked to the specialized brain areas involved in elaborating that specific experience. The hodotopic approach to phenomenology with its focus on functional anatomy and networks may be the model that best accommodates this conceptualization. Future studies will test whether this approach can be considered valid for understanding $\mathrm{VH}$ in different pathological contexts.

Supplementary Information The online version contains supplementary material available at https://doi.org/10.1007/s00415-022-10983-6.

Acknowledgements We would like to thank all the participants whose data was used in this study. We are thankful to Newcastle University, Professor Prabitha Urwyler and Professor Urs Peter Mosimann for proving us with the North East Visual Hallucinations Interview (NEVHI). The present study was partially supported by a fellowship from Joint research projects for mobility of the $\mathrm{PhD}$ fellows of Sapienza University of Rome to Fabrizia D'Antonio. DHFF thanks the National Institute for Health Research (NIHR) Mental Health Biomedical Research Centre at the South London and Maudsley NHS Foundation Trust and was supported by NIHR Programme Grants for Applied Research (RP-PG-0610-10100). The views expressed are those of the authors and not necessarily those of the NIHR or the Department of Health and Social Care.

Author contributions Conceptualization: FDA, MB, ADV, DHFF; Methodology: FDA, MB, ADV, CG, SF, DHFF; Formal analysis: FDA, MB, ADV; Investigation: FDA, ADV, AF, AS, MC; Resources: AS, FC, MF; Writing - original draft preparation: FDA; Writing — review and editing: FDA, MB, MC, CG, SF, CdL, DA, DHFF; Supervision: $\mathrm{CG}, \mathrm{CdL}, \mathrm{DA}, \mathrm{DHFF}$.

Funding No funding was received for conducting this study.

Availability of data and material The data that support the findings of this study are available from the corresponding author, upon reasonable request.

\section{Declarations}

Conflicts of interest The authors declare that they have no conflict of interest. 
Ethics approval This study was performed in line with the principles of the Declaration of Helsinki. The study was approved by Policlinico Umberto I-Sapienza University of Rome Ethics Committee (reference number: 5178).

Consent to participate Written informed consent was obtained from all participants or their caregivers prior to study participation.

Open Access This article is licensed under a Creative Commons Attribution 4.0 International License, which permits use, sharing, adaptation, distribution and reproduction in any medium or format, as long as you give appropriate credit to the original author(s) and the source, provide a link to the Creative Commons licence, and indicate if changes were made. The images or other third party material in this article are included in the article's Creative Commons licence, unless indicated otherwise in a credit line to the material. If material is not included in the article's Creative Commons licence and your intended use is not permitted by statutory regulation or exceeds the permitted use, you will need to obtain permission directly from the copyright holder. To view a copy of this licence, visit http://creativecommons.org/licenses/by/4.0/.

\section{References}

1. Aarsland D, Ballard CG, Halliday G (2004) Are Parkinson's disease with dementia and dementia with Lewy bodies the same entity? J Geriatr Psychiatry Neurol 17:137-145. https://doi.org/ $10.1177 / 0891988704267470$

2. Foguem C, Manckoundia P (2018) Lewy body disease: clinical and pathological "overlap syndrome" between synucleinopathies (Parkinson Disease) and tauopathies (Alzheimer Disease). Curr Neurol Neurosci Rep 18:24. https://doi.org/10.1007/ s11910-018-0835-5

3. Jellinger KA, Korczyn AD (2018) Are dementia with Lewy bodies and Parkinson's disease dementia the same disease? BMC Med 16:34. https://doi.org/10.1186/s12916-018-1016-8

4. Diederich NJ, Goetz CG, Stebbins GT (2005) Repeated visual hallucinations in Parkinson's disease as disturbed external/internal perceptions: focused review and a new integrative model. Mov Disord 20:130-140. https://doi.org/10.1002/mds.20308

5. Collerton D, Perry E, McKeith I (2005) Why people see things that are not there: A novel perception and attention deficit model for recurrent complex visual hallucinations. Behav Brain Sci 28:737-757. https://doi.org/10.1017/S0140525X05000130

6. Shine JM, Halliday GM, Naismith SL, Lewis SJG (2011) Visual misperceptions and hallucinations in Parkinson's disease: dysfunction of attentional control networks? Mov Disord 26:2154 2159. https://doi.org/10.1002/mds. 23896

7. Shine JM, O'Callaghan C, Halliday GM, Lewis SJG (2014) Tricks of the mind: Visual hallucinations as disorders of attention. Prog Neurobiol 116:58-65. https://doi.org/10.1016/j.pneur obio.2014.01.004

8. Shine JM, Halliday GM, Gilat M et al (2014) The role of dysfunctional attentional control networks in visual misperceptions in Parkinson's disease. Hum Brain Mapp 35:2206-2219. https:// doi.org/10.1002/hbm. 22321

9. Bronnick K, Emre M, Tekin S et al (2011) Cognitive correlates of visual hallucinations in dementia associated with Parkinson's disease. Mov Disord 26:824-829. https://doi.org/10.1002/mds. 23525

10. Hepp DH, da Hora CC, Koene T et al (2013) Cognitive correlates of visual hallucinations in non-demented Parkinson's disease patients. Park Relat Disord 19:795-799. https://doi.org/ 10.1016/j.parkreldis.2013.04.026

11. Cagnin A, Gnoato F, Jelcic N et al (2013) Clinical and cognitive correlates of visual hallucinations in dementia with Lewy bodies. J Neurol Neurosurg Psychiatry 84:505-510. https://doi. org/10.1136/jnnp-2012-304095

12. Pezzoli S, Cagnin A, Antonini A, Venneri A (2019) Frontal and subcortical contribution to visual hallucinations in dementia with Lewy bodies and Parkinson's disease. Postgrad Med 131:509-522. https://doi.org/10.1080/00325481.2019.1656515

13. Ffytche DH, Howard RJ, Brammer MJ et al (1998) The anatomy of conscious vision: an fMRI study of visual hallucinations. Nat Neurosci 1:738-742. https://doi.org/10.1038/3738

14. Ffytche DH, Creese B, Politis M et al (2017) The psychosis spectrum in Parkinson disease. Nat Rev Neurol 13:81-95. https://doi.org/10.1038/nrneurol.2016.200

15. ffytche DH, (2008) The hodology of hallucinations. Cortex 44:1067-1083. https://doi.org/10.1016/j.cortex.2008.04.005

16. Franciotti R, Delli Pizzi S, Perfetti B et al (2015) Default mode network links to visual hallucinations: A comparison between Parkinson's disease and multiple system atrophy. Mov Disord 30:1237-1247. https://doi.org/10.1002/mds.26285

17. Yao N, Shek-Kwan Chang R, Cheung C et al (2014) The default mode network is disrupted in Parkinson's disease with visual hallucinations. Hum Brain Mapp 35:5658-5666. https://doi.org/ $10.1002 / \mathrm{hbm} .22577$

18. Lenka A, Pagonabarraga J, Pal PK et al (2019) Minor hallucinations in Parkinson disease: a subtle symptom with major clinical implications. Neurology 93:259-266. https://doi.org/10.1212/ WNL.0000000000007913

19. Onofrj M, Taylor JP, Monaco D et al (2013) Visual hallucinations in PD and Lewy body dementias: old and new hypotheses. Behav Neurol 27:479-493. https://doi.org/10.3233/ BEN-129022

20. Braak H, Del Tredici K, Rub U et al (2003) Staging of brain pathology related to sporadic Parkinson's disease. Neurobiol Aging 24:197-211. https://doi.org/10.1016/s0197-4580(02) 00065-9

21. Friedman JH (2018) Dementia with lewy bodies and parkinson disease dementia: it is the same disease! Parkinsonism Relat Disord 46(Suppl 1):S6-S9. https://doi.org/10.1016/j.parkreldis.2017. 07.013

22. McKeith IG, Boeve BF, Dickson DW et al (2017) Diagnosis and management of dementia with lewy bodies: fourth consensus report of the DLB consortium. Neurology 89:88-100. https://doi. org/10.1212/WNL.0000000000004058

23. Vasconcellos LFR, Pereira JS (2015) Parkinson's disease dementia: Diagnostic criteria and risk factor review. J Clin Exp Neuropsychol 37:988-993. https://doi.org/10.1080/13803395.2015. 1073227

24. Goetz CG, Tilley BC, Shaftman SR et al (2008) Movement disorder society-sponsored revision of the Unified Parkinson's disease rating scale (MDS-UPDRS): scale presentation and clinimetric testing results. Mov Disord 23:2129-2170. https://doi.org/10. $1002 / \mathrm{mds} .22340$

25. Moller JC, Korner Y, Dodel RC et al (2005) Pharmacotherapy of Parkinson's disease in Germany. J Neurol 252:926-935. https:// doi.org/10.1007/s00415-005-0784-1

26. Woods SW (2003) Chlorpromazine equivalent doses for the newer atypical antipsychotics. J Clin Psychiatry 64:663-667. https://doi. org/10.4088/jcp.v64n0607

27. Cummings JL, Mega M, Gray K et al (1994) The Neuropsychiatric Inventory: comprehensive assessment of psychopathology in dementia. Neurology 44:2308-2314

28. Angelelli P, Paolucci S, Bivona U et al (2004) Development of neuropsychiatric symptoms in poststroke patients: a 
cross-sectional study. Acta Psychiatr Scand 110:55-63. https:// doi.org/10.1111/j.1600-0447.2004.00297.x

29. Mosimann UP, Collerton D, Dudley R et al (2008) A semi-structured interview to assess visual hallucinations in older people. Int J Geriatr Psychiatry 23:712-718. https://doi.org/10.1002/gps.1965

30. O'Brien J, Taylor JP, Ballard C et al (2020) Visual hallucinations in neurological and ophthalmological disease: pathophysiology and management. J Neurol Neurosurg Psychiatry 91:512-519. https://doi.org/10.1136/jnnp-2019-322702

31. Folstein MF, Folstein SE, McHugh PR (1975) "Mini-mental state": A practical method for grading the cognitive state of patients for the clinician. J Psychiatr Res 12:189-198. https://doi. org/10.1016/0022-3956(75)90026-6

32. Lawton MP, Brody EM (1969) Assessment of Older People: SelfMaintaining and Instrumental Activities of Daily Living1. Gerontologist 9:179-186. https://doi.org/10.1093/geront/9.3_Part_1.179

33. Carlesimo G, Buccione I, Fadda L et al (2002) Normative data of two memory tasks: Short-Story recall and Rey's Figure. Nuova Riv Neurol 12:1-3

34. Spinnler H, Tognoni G (1987) Standardizzazione e Taratura Italiana di Test Neuropsicologici. Ital J Neurol Sci Suppl 8:1-120

35. Orsini A, Grossi D, Capitani E et al (1988) Verbal and spatial immediate memory span: Normative data from 1355 adults and 1112 children. Ital J Neurol Sci 8:539-548

36. Giovagnoli AR, Del Pesce M, Mascheroni S et al (1996) Trail making test: Normative values from 287 normal adult controls. Ital J Neurol Sci 17:305-309

37. Shulman KI, Pushkar Gold D, Cohen CA, Zucchero CA (1993) Clock-drawing and dementia in the community: a longitudinal study. Int J Geriatr Psychiatry 8:487-496. https://doi.org/10.1002/ gps.930080606

38. van Gorp W, Satz P, Evans Kiersch M, Henry R (1987) Normative data on the Boston Naming Test for group of normal older adults. J Clin Exp Neuropsychol 8:702-705

39. Basso A, Capitani E, Laiacona M (1986) Raven's coloured progressive matrices: normative values on 305 adult normal controls. Funct Neurol 2:189-194

40. Appollonio I, Leone M, Isella V et al (2005) The Frontal Assessment Battery (FAB): Normative values in an Italian population sample. Neurol Sci 26:108-116

41. Warrington EK, James M (1991) The visual object and space perception battery. Thames Valley Test Company, Bury St Edmunds, England

42. Humphreys Glyn W, Riddoch JM (1993) Birmingham Object Recognition Battery (BORB). Lawrence Erlbaum Associates, Hove, UK

43. Benton AL, Varney NR, Hamsher K (1978) Visuospatial judgment: A clinical test. Arch Neurol 35:364-367

44. Whitfield-Gabrieli S, Nieto-Castanon A (2012) Conn: a functional connectivity toolbox for correlated and anticorrelated brain networks. Brain Connect 2:125-141. https://doi.org/10.1089/brain. 2012.0073

45. Power JD, Barnes KA, Snyder AZ et al (2012) Spurious but systematic correlations in functional connectivity MRI networks arise from subject motion. Neuroimage 59:2142-2154. https://doi.org/ 10.1016/j.neuroimage.2011.10.018

46. Jafri MJ, Pearlson GD, Stevens M, Calhoun VD (2008) A method for functional network connectivity among spatially independent resting-state components in schizophrenia. Neuroimage 39:16661681. https://doi.org/10.1016/j.neuroimage.2007.11.001

47. Llebaria G, Pagonabarraga J, Martínez-Corral M et al (2010) Neuropsychological correlates of mild to severe hallucinations in Parkinson's disease. Mov Disord 25:2785-2791. https://doi.org/ $10.1002 / \mathrm{mds} .23411$
48. Mori E, Shimomura T, Fujimori M et al (2000) Visuoperceptual impairment in dementia with Lewy bodies. Arch Neurol 57:489_ 493. https://doi.org/10.1001/archneur.57.4.489

49. Hamilton JM, Landy KM, Salmon DP et al (2012) Early visuospatial deficits predict the occurrence of visual hallucinations in autopsy-confirmed dementia with lewy bodies. Am J Geriatr Psychiatry 20:773-781. https://doi.org/10.1097/JGP.0b013e3182 $3033 \mathrm{bc}$

50. Ishioka T, Hirayama K, Hosokai Y et al (2011) Illusory misidentifications and cortical hypometabolism in Parkinson's disease. Mov Disord 26:837-843. https://doi.org/10.1002/mds.23576

51. Imamura K, Wada-Isoe K, Kitayama M, Nakashima K (2008) Executive dysfunction in non-demented Parkinson's disease patients with hallucinations. Acta Neurol Scand 117:255-259. https://doi.org/10.1111/j.1600-0404.2007.00933.x

52. Shin S, Lee JE, Hong JY et al (2012) Neuroanatomical substrates of visual hallucinations in patients with non-demented Parkinson's disease. J Neurol Neurosurg Psychiatry 83:1155-1161. https://doi. org/10.1136/jnnp-2012-303391

53. Muller AJ, O'Callaghan C, Walton CC et al (2017) Retrospective Neuropsychological Profile Of Patients with Parkinson disease prior to developing visual hallucinations. J Geriatr Psychiatry Neurol 30:90-95. https://doi.org/10.1177/0891988716686830

54. Tosi G, Borsani C, Castiglioni S et al (2020) Complexity in neuropsychological assessments of cognitive impairment: A network analysis approach. Cortex 124:85-96. https://doi.org/10.1016/j. cortex.2019.11.004

55. D'Antuono G, La Torre FR, Marin D et al (2017) Role of working memory, inhibition, and fluid intelligence in the performance of the Tower of London task. Appl Neuropsychol Adult 24:548-558. https://doi.org/10.1080/23279095.2016.1225071

56. Duncan J (1995) Attention, intelligence, and the frontal lobes. In: Gazzaniga MS (ed) The cognitive neurosciences. MIT Press, Cambridge, MA, pp 721-733

57. Duncan J, Burgess P, Emslie H (1995) Fluid intelligence after frontal lobe lesions. Neuropsychologia 33:261-268. https://doi. org/10.1016/0028-3932(94)00124-8

58. Grill-Spector K, Kourtzi Z, Kanwisher N (2001) The lateral occipital complex and its role in object recognition. Vision Res 41:1409-1422. https://doi.org/10.1016/s0042-6989(01)00073-6

59. Aminoff EM, Kveraga K, Bar M (2013) The role of the parahippocampal cortex in cognition. Trends Cogn Sci 17:379-390. https://doi.org/10.1016/j.tics.2013.06.009

60. Levy I, Hasson U, Avidan G et al (2001) Center-periphery organization of human object areas. Nat Neurosci 4:533-539. https://doi. org/10.1038/87490

61. Mikellidou K, Kurzawski JW, Frijia F et al (2017) Area Prostriata in the Human Brain. Curr Biol 27:3056-3060.e3. https://doi.org/ 10.1016/j.cub.2017.08.065

62. Sergent J (1982) The cerebral balance of power: confrontation or cooperation? J Exp Psychol Hum Percept Perform 8:253-272. https://doi.org/10.1037//0096-1523.8.2.253

63. Pagonabarraga J, Soriano-Mas C, Llebaria G et al (2014) Neural correlates of minor hallucinations in non-demented patients with Parkinson's disease. Park Relat Disord 20:290-296. https://doi. org/10.1016/j.parkreldis.2013.11.017

64. Janzen J, Van'T Ent D, Lemstra AW, et al (2012) The pedunculopontine nucleus is related to visual hallucinations in Parkinson's disease: Preliminary results of a voxel-based morphometry study. J Neurol 259:147-154. https://doi.org/10.1007/ s00415-011-6149-Z

65. Nishio Y, Yokoi K, Uchiyama M et al (2017) Deconstructing psychosis and misperception symptoms in Parkinson's disease. J Neurol Neurosurg Psychiatry 88:722-729. https://doi.org/10. 1136/jnnp-2017-315741 
66. Hepp DH, Foncke EMJ, Olde Dubbelink KTE et al (2017) Loss of Functional Connectivity in Patients with Parkinson Disease and Visual Hallucinations. Radiology 285:896-903. https://doi.org/ 10.1148/radiol.2017170438

67. Thiebaut de Schotten M, Dell'Acqua F, Forkel SJ et al (2011) A lateralized brain network for visuospatial attention. Nat Neurosci 14:1245-1246. https://doi.org/10.1038/nn.2905

68. Zorzi G, Thiebaut de Schotten M, Manara R et al (2021) White matter abnormalities of right hemisphere attention networks contribute to visual hallucinations in dementia with Lewy bodies. Cortex 139:86-98. https://doi.org/10.1016/j.cortex.2021.03.007

69. Seghier ML (2013) The angular gyrus: multiple functions and multiple subdivisions. Neuroscientist 19:43-61. https://doi.org/ $10.1177 / 1073858412440596$

70. Iaccarino L, Sala A, Caminiti SP et al (2018) The brain metabolic signature of visual hallucinations in dementia with Lewy bodies. Cortex 108:13-24. https://doi.org/10.1016/j.cortex.2018.06.014

71. Peraza LR, Kaiser M, Firbank M et al (2014) fMRI resting state networks and their association with cognitive fluctuations in dementia with Lewy bodies. NeuroImage Clin 4:558-565. https:// doi.org/10.1016/j.nicl.2014.03.013

72. Lobotesis K, Fenwick JD, Phipps A et al (2001) Occipital hypoperfusion on SPECT in dementia with Lewy bodies but not AD. Neurology 56:643-649. https://doi.org/10.1212/wnl.56.5.643

73. Colloby SJ, Fenwick JD, Williams ED et al (2002) A comparison of $(99 \mathrm{~m}) \mathrm{Tc}-\mathrm{HMPAO}$ SPET changes in dementia with Lewy bodies and Alzheimer's disease using statistical parametric mapping. Eur J Nucl Med Mol Imaging 29:615-622. https://doi.org/10. 1007/s00259-002-0778-5

74. Teune LK, Bartels AL, de Jong BM et al (2010) Typical cerebral metabolic patterns in neurodegenerative brain diseases. Mov Disord 25:2395-2404. https://doi.org/10.1002/mds.23291
75. Ffytche DH (2007) Visual hallucinatory syndromes: past, present, and future. Dialogues Clin Neurosci 9:173-189

76. Perry EK, Perry RH (1995) Acetylcholine and hallucinations: disease-related compared to drug-induced alterations in human consciousness. Brain Cogn 28:240-258. https://doi.org/10.1006/ brcg.1995.1255

77. Handjaras G, Ricciardi E, Szczepanik J et al (2013) Cholinergic enhancement differentially modulates neural response to encoding during face identity and face location working memory tasks. Exp Biol Med (Maywood) 238:999-1008. https://doi.org/10.1177/ 1535370213497326

78. Orsolini L, Papanti GD, De Berardis D et al (2017) The "Endless Trip" among the NPS Users: Psychopathology and Psychopharmacology in the Hallucinogen-Persisting Perception Disorder. A Systematic Review Front psychiatry 8:240. https://doi.org/10. 3389/fpsyt.2017.00240

79. McGuire PK, Cope H, Fahy TA (1994) Diversity of psychopathology associated with use of 3,4-methylenedioxymethamphetamine ('Ecstasy'). Br J Psychiatry 165:391-395. https://doi.org/10.1192/ bjp.165.3.391

80. Nichols DE (2004) Hallucinogens. Pharmacol Ther 101:131-181. https://doi.org/10.1016/j.pharmthera.2003.11.002

81. Merlau-Ponty (1945) Phénoménologie de la Perception. Gallimard, Paris

Publisher's Note Springer Nature remains neutral with regard to jurisdictional claims in published maps and institutional affiliations. 